\title{
Fault Tolerant Control approach based on Multiple Models and Set-Membership State estimation
}

\author{
S. Ben Chabane, C. Stoica Maniu, E.F. Camacho, T. Alamo, D. Dumur
}

\begin{abstract}
This paper proposes a new Fault Tolerant Control technique based on the Multiple Models approach for linear systems with bounded perturbations and measurement noises. The consistency of each model with the measurements is checked at each sample time, using an ellipsoidal set-membership state estimation. A Min-Max Model Predictive Control is developed in order to find the optimal control and the best model in spite of the simultaneous presence of component and/or actuator and/or sensor faults. An illustrative example is analyzed in order to show the effectiveness of the proposed approach.

Index Terms-FTC, FD, Multiple Models, set-membership state estimation, Min-Max MPC, uncertain linear systems.
\end{abstract}

\section{INTRODUCTION}

In the literature, Fault Detection approaches can be categorized into stochastic approaches (stochastic uncertainties) and deterministic approaches (bounded uncertainties by compact set). In general, a deviation of at least one characteristic property or parameter of a system from its acceptable/usual/standard conditions is considered as a fault. The determination of a fault at a certain time is referred to Fault Detection (FD) [1], [2]. Fault Tolerant Control (FTC) is a relatively new research area that makes possible the development of control laws which allow us to maintain current performances close to desirable objectives even after the occurrence of faults. A general technique used in the literature consists in designing a Fault Tolerant Controller that can adapt or reconfigure itself based on the FD information such that the system can still operate safely despite the presence of faults. There are three parts of a system susceptible to faults: actuators, system's components and sensors.

One of the many different approaches of FD is the Multiple Models (MM) technique. A Multiple Model technique consists in the construction of a set of models that contains local information corresponding to specific fault conditions of the monitored system [3], [4]. The motivation for using Multiple Model systems for FD stems from the fact that a large class of fault conditions can be modeled simultaneously, contrary to other FD methods that can only be applied to limited types/number of fault conditions (e.g. actuator or sensor faults). In addition, the use of Multiple linear Models

S. Ben Chabane, C. Stoica Maniu and D. Dumur are with Laboratoire des Signaux et Systèmes, CentraleSupélec-CNRS-Univ. Paris-Sud, Université Paris Saclay, 3 rue Joliot Curie, F-91192, Gif-sur-Yvette cedex, France (e-mail: \{sofiane.benchabane; cristina.stoica; didier.dumur\}asupelec.fr).

T. Alamo and E.F. Camacho are with Department of Ingeniería de Sistemas y Automática, Universidad de Sevilla, Camino de los Descubrimientos, 41092 Sevilla, Spain (e-mail: alamodcartuja.us.es, eduardo@esi.us.es). represents an attractive solution to deal with the control of non-linear systems [5], [6], [7], [8], [9]. This is motivated by the fact that non-linear systems can be modeled by Linear Parametric Varying (LPV) models [10], [11], Takagi-Sugeno fuzzy models [12], [13], [14], etc.

Multiple Model systems are also used in the context of FD for linear systems due to its flexibility and simplicity, which allows us an intuitive modeling of faults. The authors of [15] propose a method for estimating both the weights and the state of a Multiple Model system with one common state vector. In this system, the weights are related to the activation of each individual model. Perturbations and measurement noises are assumed to be stochastic with a given covariance representation. Paper [16] presents a different fault diagnosis method based on a generation of the residuals. These residual signals are obtained in a statistical framework which sometimes makes difficult the parameters tuning. Generally, in the stochastic methods, the perturbations are assumed to have a known distribution. This assumption is in many cases difficult to validate. Thus, it may be more realistic to assume that the perturbations and measurement noises are unknown but bounded. This leads to use set-membership approaches for the estimation [17], [18], [19], [20].

In this context, the current paper proposes a new Fault Tolerant Control method (using set-membership state estimation) based on Multiple Models technique. These models are constructed by referring to the original system, such that each model is adequate to one faulty mode. This method consists first in checking the consistency between each model with the available measurements. The consistency test is based on a guaranteed ellipsoidal set-membership state estimation [21]. Second, the set of compatible models with the measurements is formed. In a third step, a Min-Max Model Predictive Control (MPC) [22] is developed for each compatible model ensuring the desirable performances. A quadratic criterion is minimized in order to choose the best control to be applied to the original system and the best model for the estimation.

The novelty of this paper is the use of set-membership estimation coupled with Min-Max MPC to estimate the state of linear systems with unknown but bounded perturbations and measurement noises despite the simultaneous presence of component, actuator and sensor faults.

Notations: An interval $[a, b]$ is defined by the set $\{x \in \mathbb{R}: a \leq x \leq b\}$. A unitary interval is $\mathbf{B}=[-1,1]$. A box $\left(\left[a_{1}, b_{1}\right], \ldots,\left[a_{n}, b_{n}\right]\right)^{\top}$ is an interval vector. A unitary box in $\mathbb{R}^{m}$, denoted by $\mathbf{B}^{m}$, is a box composed by $m$ unitary intervals. A bounded ellipsoidal set $\mathcal{E}(P, \bar{x}, \rho)$ is defined by 
$\mathcal{E}(P, \bar{x}, \rho)=\left\{x \in \mathbb{R}^{n}:(x-\bar{x})^{\top} P(x-\bar{x}) \leq \rho\right\}$, where $P=P^{\top} \succ 0$ is the shape matrix of the ellipsoid, $\bar{x} \in \mathbb{R}^{n_{x}}$ is its center and $\rho \in \mathbb{R}_{+}^{*}$ is its radius. A polyhedron $\mathcal{P} \in \mathbb{R}^{n}$ is defined by a system of finitely many inequalities $A x \leq b$ such that $\mathcal{P}=\left\{x \in \mathbb{R}^{n}: A x \leq b\right\}$. Given a bounded polyhedral set $\mathcal{X}$, denote by $\mathcal{V}_{\mathcal{X}}$ the set of its vertices. A polytope $\mathcal{P} \in \mathbb{R}^{n}$ is defined by a finite set $\mathcal{X} \subseteq \mathbb{R}^{n}$ such that $\mathcal{P}=\operatorname{conv}(\mathcal{X})$. A strip is defined by $\mathcal{S}(y, c, \sigma)=\left\{x \in \mathbb{R}^{n}\right.$ : $\left.\left|c^{\top} x-y\right| \leq \sigma\right\}$. The symbol $\|\cdot\|_{1}$ denotes the norm 1 . Denote by $\mathcal{C}_{M}$ the set of compatible models with the measurements. The matrices $\mathbb{O}_{n, m}, \mathbb{\nabla}_{n}$ and $\mathbb{1}_{n, m}$ denotes respectively a zeros matrix in $\mathbb{R}^{n \times m}$, an identity matrix in $\mathbb{R}^{n \times n}$ and a matrix in $\mathbb{R}^{n \times m}$ having all elements equal to 1 .

\section{PRoblem FORMUlation}

Consider the following discrete-time LTI (Linear Time Invariant) system:

$$
\left\{\begin{array}{l}
x_{k+1}=A G_{i_{c}} x_{k}+B H_{i_{a}} u_{k}+E \omega_{k} \\
y_{k}=C I_{i_{s}} x_{k}+F \omega_{k}
\end{array}\right.
$$

with $A \in \mathbb{R}^{n_{x} \times n_{x}}, B \in \mathbb{R}^{n_{x} \times n_{u}}, C \in \mathbb{R}^{n_{y} \times n_{x}}, E \in$ $\mathbb{R}^{n_{x} \times\left(n_{x}+n_{y}\right)}, F \in \mathbb{R}^{n_{y} \times\left(n_{x}+n_{y}\right)}, G_{i_{c}} \in \mathbb{R}^{n_{x} \times n_{x}}, H_{i_{a}} \in$ $\mathbb{R}^{n_{u} \times n_{u}}$ and $I_{i_{s}} \in \mathbb{R}^{n_{x} \times n_{x}}$. Here, $x_{k} \in \mathbb{R}^{n_{x}}$ is the state vector of the system, $u_{k} \in \mathbb{R}^{n_{u}}$ is the input vector, and $y_{k} \in \mathbb{R}^{n_{y}}$ is the measured output vector at sample time $k$. The vector $\omega_{k}$ contains the state perturbations and the measurement perturbations (noise, offset, etc.), which are assumed to be bounded by unitary boxes $\omega_{k} \in \mathbf{B}^{n_{x}+n_{y}}$ for every $k \geq 0$. Consider that the initial state $x_{0}$ belongs to the ellipsoid $\mathcal{E}\left(P_{0}, \bar{x}_{0}, \rho_{0}\right)=\left\{x \in \mathbb{R}^{n_{x}}:\left(x-\bar{x}_{0}\right)^{\top} P_{0}(x-\right.$ $\left.\left.\bar{x}_{0}\right) \leq \rho_{0}\right\}$.

The matrix $G_{i_{c}}$, with $i_{c} \in \mathbb{\mathbb { V }}_{c}=\left\{0,1,2, \ldots, n_{c}\right\}$ and $n_{c}$ denoting the number of the considered component faults, is a diagonal matrix modeling the $i_{c}$-th component mode. In a similar way, the matrix $H_{i_{a}}$, with $i_{a} \in \mathbb{\mathbb { V }}_{a}=\left\{0,1,2, \ldots, n_{a}\right\}$ and $n_{a}$ the number of considered actuator faults, is a diagonal matrix modeling the $i_{a}$-th actuator mode. The matrix $I_{i_{s}}$, with $i_{s} \in \mathbb{\nabla}_{s}=\left\{0,1,2, \ldots, n_{s}\right\}$, where $n_{s}$ denotes the number of considered sensor faults, is a diagonal matrix modeling the $i_{s}$-th sensor mode.

All diagonal entries of $G_{i_{c}}, H_{i_{a}}$ and $I_{i_{s}}$ belong to $(0,1]$ where 0 or 1 means that the corresponding components, actuators and sensors are completely faulty or healthy, respectively. A value in the range $(0,1)$ denotes a partial degradation of the corresponding components/actuators/sensors.

Remark 1: The system (1) can be rewritten such as

$$
\left\{\begin{array}{l}
x_{k+1}=A\left(x_{k}+f_{x_{k}}\right)+B\left(u_{k}+f_{u_{k}}\right)+E \omega_{k} \\
y_{k}=C x_{k}+F \omega_{k}+f_{y_{k}}
\end{array}\right.
$$

where $f_{x_{k}}, f_{u_{k}}$ and $f_{y_{k}}$ are respectively the component fault, actuator fault and the sensor fault. It is easy to verify this, by taking $f_{x_{k}}=\left(G_{i_{c}}-\square_{n_{x}}\right) x_{k}, f_{u_{k}}=\left(H_{i_{a}}-\square_{n_{u}}\right) u_{k}$ and $f_{y_{k}}=\left(I_{i_{s}}-\mathbb{\square}_{n_{x}}\right) x_{k}$.

Given an ellipsoidal estimation for $x_{k}$ of the form $\mathcal{E}\left(P, \bar{x}_{k}, \rho_{k}\right)$, with $P$ unknown and $k>0$, the objective of this paper is to provide an ellipsoidal estimation for $x_{k+1}$ of the form $\mathcal{E}\left(P, \bar{x}_{k+1}, \rho_{k+1}\right)$ using the ellipsoidal setmembership state estimation presented in [21] despite the presence of possible faults ${ }^{1}$ on components, actuators and/or sensors.

\section{Multiple Models Fault Tolerant Control}

First of all, a set of $p$ Multiple Models $\mathcal{M}=$ $\left\{M_{1}, M_{2}, \ldots, M_{p}\right\}$ is further constructed such that $M_{1}$ represents the fault-free case, i.e. $A_{1}=A, B_{1}=B, C_{1}=C$, $E_{1}=E$ and $F_{1}=F$. Then, for $i=2, \ldots, p$, each model $M_{i}$ is dedicated to one faulty mode. Note that the model $M_{i}$ is defined by the matrices $A_{i}=A G_{i_{c}}, B_{i}=B H_{i_{a}}$, $C_{i}=C I_{i_{s}}, E_{i}=E$ and $F_{i}=F$, for $i=1, \ldots, p$. A good knowledge of the system is required in order to define these $p$ models

$$
\left\{\begin{array}{l}
x_{k+1}=A_{i} x_{k}+B_{i} u_{k}+E_{i} \omega_{k} \\
y_{k}=C_{i} x_{k}+F_{i} \omega_{k}
\end{array}, i=1, \ldots, p\right.
$$

The state of the system (1) is estimated in parallel by each model $M_{i}$ based on the ellipsoidal estimation developed in [21] for the fault-free case.

Remark 2: For each model $M_{i}$, the system matrices $A$, $B$ and $C$ from [21] are replaced by $A G_{i_{c}}, B H_{i_{a}}$ and $C I_{i_{s}}$ respectively, in order to estimate the state of the system by solving the LMI problem (16) of [21]. This LMI problem will be denoted by $(16)^{*}$ in the rest of the present paper.

Considering the presence of faults, the consistency between the model $M_{i}$ and the measurement has to be checked at each sample time. Thus, the objective is to find the models which are compatible with the set of measurements. Once this set is computed, a Min-Max Model Predictive Control is developed in order to stabilize the state $x_{k}$ of the system (1) and to decide which is the best model to estimate the state of the system for the next step. The details of the Min-Max MPC problem are given in Section IV.

Algorithm 1 provides a general form of the Fault Detection and Fault Tolerant Control strategy based on checking consistency between the models and the measurements.

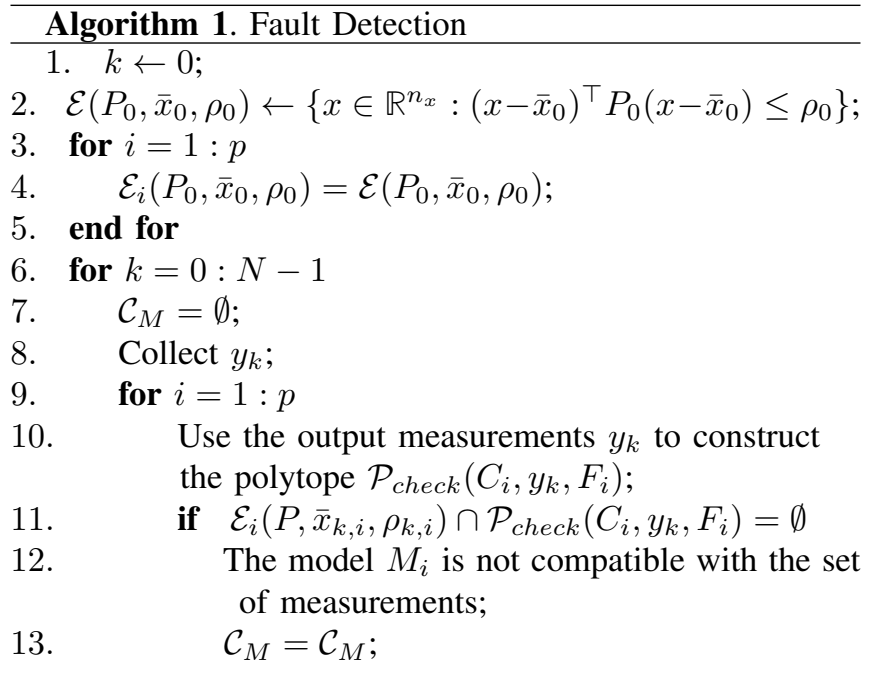

\footnotetext{
${ }^{1}$ Note that other existing estimation techniques could be applied, e.g.
} zonotopic state estimation [23], [24], ellipsoidal state estimation [25], [19]. 
14.

15.

16.

17.

18.

19.

20.

21.

22.

23.

24.

25.

26.

27.

28.

29.

30.

31.

32.

33. Compute the ellipsoidal estimation set

model

end if

end for

$s_{M}=\operatorname{size}\left(\mathcal{C}_{M}\right)$

$\mathcal{U}_{k}=\emptyset$

for $j=1: s_{M}$

Compute $\mathbf{u}_{k \mid k, j}$ by solving the criterion (6);

$\mathcal{U}_{k}=\left\{\mathcal{U}_{k}, \mathbf{u}_{k \mid k, j}\right\}$

end for

Compute $u_{k \mid k}^{*}$ and $M_{k}^{*}$ using (7) and (8);

for $i=1: p$

if $M_{i} \in \mathcal{C}_{\mathcal{M}}$

Compute the ellipsoidal estimation set

$\mathcal{E}_{i}\left(P, \bar{x}_{k+1, i}, \rho_{k+1, i}\right)$ according to $(16)^{*}$ using the model $M_{i}$ (defined by the matrices $A_{i}$, else $B_{i}, C_{i}, E_{i}$ and $\left.F_{i}\right)$ and the control $u_{k \mid k}^{*}$;

Compute the ellipsoidal estimation set

$\mathcal{E}_{i}\left(P, \bar{x}_{k+1, i}, \rho_{k+1, i}\right)$ according to $(16)^{*}$ using end if

$M_{k}^{*}$ and $u_{k \mid k}^{*}$;

\section{end for}

$\mathcal{E}\left(P, \bar{x}_{k+1}, \rho_{k+1}\right)$ according to $(16)^{*}$ using the

$M_{k}^{*}$

34. $k=k+1$

35. end for.

This algorithm is summarized below:

- Initialization: (steps 1 to 5)

The estimated state is initialized by the ellipsoidal set $\mathcal{E}\left(P_{0}, \bar{x}_{0}, \rho_{0}\right)$ in step 2 . The estimation set for each model $M_{i} \in \mathcal{M}$, with $i=1, \ldots, p$, is also initialized by the same ellipsoidal set $\mathcal{E}_{i}\left(P_{0}, \bar{x}_{0}, \rho_{0}\right)=\mathcal{E}\left(P_{0}, \bar{x}_{0}, \rho_{0}\right)$. These ellipsoids are chosen sufficiently large in order to contain the real initial state.

- Compatible models set construction: (steps 7 to 18) At each sample time $k$, the output measurement $y_{k}$ in (1) obtained from the sensors is used to build the parametrized polytope ${ }^{2} \mathcal{P}_{\text {check }}\left(C_{i}, y_{k}, F_{i}\right)$ for each model $M_{i}$, with $i=1, \ldots, p$. This polytope corresponds to the consistent state set with the measurements $y_{k}$. The construction of the polytope $\mathcal{P}_{\text {check }}\left(C_{i}, y_{k}, F_{i}\right)$ is obtained from the intersection of all the $n_{y}$ measurement strips $\mathcal{S}_{j}\left(y_{k, j}, C_{i, j},\left\|F_{i, j}\right\|_{1}\right)$ (each strip is formed by one of the $n_{y}$ scalar components $y_{k, j}$ of the vector $y_{k}$, with $\left.j=1, \ldots, n_{y}\right)$. Each strip is defined by these

\footnotetext{
${ }^{2}$ For Single Output systems, a measurement strip is used. For MultiOutput systems, intersecting all the measurement strips related to each scalar component of the output leads to a polytope.
}

two inequalities

$$
\left\{\begin{array}{l}
C_{i, j} x_{k} \leq y_{k, j}+\left\|F_{i, j}\right\|_{1} \\
-C_{i, j} x_{k} \leq-y_{k, j}+\left\|F_{i, j}\right\|_{1}
\end{array}\right.
$$

with $i=1, \ldots, p$ and $j=1, \ldots, n_{y}$, such that $i$ represents the $i^{\text {th }}$ model and $j$ represents the $j^{\text {th }}$ line of $C_{i}, F_{i}$ and $y_{k}$ in (3).

Then, using (4), the polytope $\mathcal{P}_{\text {check }}\left(C_{i}, y_{k}, F_{i}\right)$ is defined by the following constraints

$$
\mathcal{P}_{\text {check }}\left(C_{i}, y_{k}, F_{i}\right)=\left\{x_{k} \in \mathbb{R}^{n_{x}}: S x_{k} \leq T\right\},
$$

with the matrices $S=\left[\begin{array}{c}C_{i} \\ -C_{i}\end{array}\right], T=\left[\begin{array}{c}y_{k}+\mathbf{F}_{i} \\ -y_{k}+\mathbf{F}_{i}\end{array}\right]$ and $\mathbf{F}_{i}=\left[\begin{array}{c}\left\|F_{i, 1}\right\|_{1} \\ \vdots \\ \left\|F_{i, n_{y}}\right\|_{1}\end{array}\right]$. Note that $F_{i, j}$ represents the $j^{\text {th }}$ line of the $F_{i}$ matrix for the $M_{i}$ model.

The consistency between the ellipsoidal estimated set $\mathcal{E}_{i}\left(P, \bar{x}_{k, i}, \rho_{k, i}\right)$ and the polytope $\mathcal{P}_{\text {check }}\left(C_{i}, y_{k}, F_{i}\right)$ is verified for each model $M_{i} \in \mathcal{M}$, with $i=1, \ldots, p$. The ellipsoidal set $\mathcal{E}_{i}\left(P, \bar{x}_{k, i}, \rho_{k, i}\right)$ represents the state estimation with the model $M_{i}$.

This consistency test (i.e. the intersection between an ellipsoid and a polytope) is solved by the following Quadratic Programming (QP) optimization problem with linear constraints:

$\rho_{k}^{*}=\min _{x_{k} \in \mathcal{E}_{i}\left(P, \bar{x}_{k}, \rho_{k}\right)}\left(x_{k}-\bar{x}_{k}\right)^{\top} P\left(x_{k}-\bar{x}_{k}\right)$

subject to

$$
S x_{k} \leq T .
$$

If $\rho_{k}^{*}<\rho_{k}$, then the intersection $\mathcal{E}_{i}\left(P, \bar{x}_{k, i}, \rho_{k, i}\right) \cap$ $\mathcal{P}_{\text {check }}\left(C_{i}, y_{k}, F_{i}\right)$ is not empty, the model $M_{i}$ is called compatible with the measurements and it is added to the set $\mathcal{C}_{M}$ containing all the compatible models with the measurements. Otherwise, the intersection is empty, i.e. $\mathcal{E}_{i}\left(P, \bar{x}_{k, i}, \rho_{k, i}\right) \cap \mathcal{P}_{\text {check }}\left(C_{i}, y_{k}, F_{i}\right)=\emptyset$ and the model $M_{i}$ is called incompatible with the measurements. This process is repeated for each model $M_{i}$, with $i=$ $1, \ldots, p$.

Remark 3: Note that several models $M_{i}$ of $\mathcal{M}$ can be compatible with the measurement at the same time.

- Designing a Min-Max Model Predictive Control for each compatible model: (steps 19 to 24)

A Min-Max Model Predictive Control is developed for each model compatible with the measurement. This control can be used for stabilizing a system for example by satisfying constraints on the state and the control signals. A Min-Max optimization problem is solved in order to minimize a quadratic criterion for the worstcase perturbations belonging in a bounded compact set. This corresponds for instance to the energy minimization for the worst case considered perturbations.

In order to obtain the set of controllers suitable for each model, a control sequence $\mathbf{u}_{k \mid k, j}=$ $\left[u_{k \mid k, j}, u_{k+1 \mid k, j}, \ldots, u_{k+h-1 \mid k, j}\right]^{\top}$ is computed for 
each model $M_{j} \in \mathcal{C}_{M}$, with $j=1, \ldots, s_{M}$ (where $s_{M}$ is the size of $\mathcal{C}_{M}$ ), by minimizing the criterion

$$
\mathbf{u}_{k \mid k, j}=\arg \min _{\mathbf{u}_{k \mid k, j}} \max _{\omega_{k} \in \mathbf{B}^{n_{x}+n_{y}}} J_{j}\left(u_{k \mid k, j}, \omega_{k \mid k, j}, x_{k \mid k, j}\right),
$$

subject to

$$
\begin{aligned}
& x_{k+l \mid k} \in \mathbb{X} \text { for } l=1, \ldots, h \\
& u_{k+l \mid k} \in \mathbb{U} \text { for } l=1, \ldots, h
\end{aligned}
$$

where $h$ is the prediction horizon, $x_{k+l \mid k}, u_{k+l \mid k}$ and $\omega_{k+l \mid k}$ represent the prediction of the state, the control prediction and the perturbation prediction for the sample time $k+l$ at the sample time $k$. The cost function is defined by $J_{j}\left(u_{k \mid k, j}, \omega_{k \mid k, j}, x_{k \mid k, j}\right)=$ $\sum_{l=0}^{h-1}\left(x_{k+l+1 \mid k, j}^{\top} Q x_{k+l+1 \mid k, j}+u_{k+l \mid k, j}^{\top} R u_{k+l \mid k, j}\right)$. The index $j$ refers to the model $M_{j} \in \mathcal{C}_{M}$, with $j=1, \ldots, s_{M}$. Generally, the constraints on the state and input vectors and the choice of the weighting matrices $Q$ and $R$ are due to physical limitations, safety and/or performance considerations. Then, the set of controllers $\mathcal{U}_{k}=\left\{\mathbf{u}_{k \mid k, 1}, \ldots, \mathbf{u}_{k \mid k, s_{M}}\right\}$ suitable for each model $M_{j} \in \mathcal{C}_{M}$ is constructed. More details on solving the problem (6) are given in Section IV.

- Computing the optimal control and the best model for the estimation: (step 25)

The objective is to determine the best control $\mathbf{u}_{k \mid k, j}^{*} \in$ $\mathcal{U}_{k}$ for the system in a faulty situation (1) and the best model $M_{j}^{*}=M_{k \mid k}^{*} \in \mathcal{C}_{M}$ to use for the estimation in case of faults. For this, the following optimization problem is solved

$$
\left(\mathbf{u}_{k \mid k}^{*}, M_{k \mid k}^{*}\right)=\arg \min _{\mathbf{u}_{k \mid k} \in \mathcal{U}_{k}} \max _{M_{j} \in \mathcal{C}_{M}} J\left(u_{k \mid k}, \omega_{k \mid k}, x_{k \mid k}\right),
$$

with the cost function $J\left(u_{k \mid k}, \omega_{k \mid k}, x_{k \mid k}\right)=$ $\sum_{l=0}^{h-1}\left(x_{k+l+1 \mid k}^{\top} Q x_{k+l+1 \mid k}+u_{k+l \mid k}^{\top} R u_{k+l \mid k}\right)$.

Based on the receding horizon strategy, the control $u_{k \mid k}^{*}$ that will be applied to the system (1) is given by the first $n_{u}$ components of the control sequence $\mathbf{u}_{k \mid k}^{*}$ as follows

$$
u_{k \mid k}^{*}=\left[\begin{array}{ll}
\mathbb{n}_{n_{u}} & \mathbb{O}_{n_{u},(h-1) n_{u}}
\end{array}\right] \mathbf{u}_{k \mid k}^{*} .
$$

- Computing the estimation for each model: (steps 26 to 32)

Each model $M_{i} \in \mathcal{M}$, with $i=1, \ldots, p$, must be fed with an ellipsoidal estimation set that will be used to construct the new set of compatible models $\mathcal{C}_{M}$ at the next sample time $k$. It consists in computing the ellipsoidal estimation sets $\mathcal{E}_{i}\left(P, \bar{x}_{k+1, i}, \rho_{k+1, i}\right)$ for each model $M_{i} \in \mathcal{M}$, with $i=1, \ldots, p$. If the model $M_{i}$ was compatible with the measurement $y_{k}$ (i.e. $M_{i} \in \mathcal{C}_{M}$ ), then the ellipsoidal estimation set $\mathcal{E}_{i}\left(P, \bar{x}_{k+1, i}, \rho_{k+1, i}\right)$ is computed according to $(16)^{*}$ using the model $M_{i}$, the control $u_{k \mid k}^{*}$ and the measurement $y_{k}$. Otherwise, the ellipsoidal estimation set $\mathcal{E}_{i}\left(P, \bar{x}_{k+1, i}, \rho_{k+1, i}\right)$ is computed according to $(16)^{*}$ using the best model $M_{k}^{*}$, the control $u_{k \mid k}^{*}$ and the measurement $y_{k}$, in order to offer a state estimation for this model which is incompatible with the measurement.

- Obtaining the final estimation: (step 33)

Finally, at time $k+1$, the ellipsoidal estimation set $\mathcal{E}\left(P, \bar{x}_{k+1}, \rho_{k+1}\right)$ is based on the best model $M_{k}^{*}$, the optimal control $u_{k \mid k}^{*}$ and $y_{k}$.

\section{Min-Max Model Predictive Control}

This section details the development of Min-Max Model Predictive Control applied to each model $M_{j}$, with $j=$ $1, \ldots, s_{M}$, belonging to the compatible set $\mathcal{C}_{M}$. The control signal is found by minimizing a worst case (with respect to the perturbations $\omega_{k}$ ) of a quadratic criterion (6). The Min-Max optimization problem (6) is reformulated as a quadratic programming $(\mathrm{QP})$ problem. Then, the controller is designed using the ellipsoidal state estimation from the previous sample time by solving a QP problem.

Starting from the quadratic cost function with simplified notations $^{3}$

$$
J_{j}\left(u_{k, j}, \omega_{k}, x_{k, j}\right)=\sum_{l=0}^{h-1}\left(x_{k+l, j}^{\top} Q x_{k+l, j}^{\top}+u_{k+l, j}^{\top} R u_{k+l, j}\right)
$$

the following state equations are computed for each compatible model $M_{j} \in \mathcal{C}_{M}$, with $j=1, \ldots, s_{M}$

$$
\left\{\begin{aligned}
x_{k+1, j}= & A_{j} x_{k, j}+B_{j} u_{k, j}+E_{j} \omega_{k, j} \\
\vdots & \\
x_{k+l, j}= & A_{j}^{l} x_{k, j}+A_{j}^{l-1} B_{j} u_{k, j}+A_{j}^{l-2} B_{j} u_{k+1, j}+ \\
& +\ldots+B_{j} u_{k+l-1, j}+A_{j}^{l-1} F_{j} \omega_{k, j}+ \\
& +A_{j}^{l-2} F_{j} \omega_{k+1, j}+\ldots+F_{j} \omega_{k+l-1, j} \\
\vdots & \\
x_{k+h, j}= & A_{j}^{h} x_{k, j}+A_{j}^{h-1} B_{j} u_{k, j}+A_{j}^{h-2} B_{j} u_{k+1, j}+ \\
& +\ldots+B_{j} u_{k+h-1, j}+A_{j}^{h-1} F_{j} \omega_{k, j}+ \\
& +A_{j}^{h-2} F_{j} \omega_{k+1, j}+\ldots+F_{j} \omega_{k+h-1, j}
\end{aligned}\right.
$$

with $h$ the prediction horizon. Denote by $\mathbf{u}_{k \mid k, j}=$ $\left[u_{k \mid k, j}, u_{k+1 \mid k, j}, \ldots, u_{k+h-1 \mid k, j}\right]^{\top} \quad$ and $\quad \boldsymbol{\omega}_{k \mid k, j}=$ $\left[\omega_{k \mid k, j}, \omega_{k+1 \mid k, j}, \ldots, \omega_{k+h-1 \mid k, j}\right]^{\top}$ the sequences of control signals and perturbations, respectively. Then, the state equation predicted for time $k+l$ at time $k$ of the model $M_{j} \in \mathcal{C}_{M}$ can be rewritten as

$$
x_{k+l \mid k, j}=A_{j}^{l} x_{k \mid k, j}+\mathcal{A}_{l, j} B_{j} \mathbf{u}_{k \mid k, j}+\mathcal{A}_{l, j} F_{j} \boldsymbol{\omega}_{k \mid k, j}
$$

where the $\mathcal{A}_{l, j}$ matrix is defined by

$$
\mathcal{A}_{l, j}=\left[\begin{array}{lllll}
A_{j}^{l-1} & A_{j}^{l-2} & \ldots & A_{j}^{0} & Z_{l}
\end{array}\right]
$$

with $Z_{l}=\underbrace{\left[\begin{array}{lll}\mathbb{O}_{n_{x}, n_{x}} & \cdots & \mathbb{O}_{n_{x}, n_{x}}\end{array}\right]}_{h-j \text { times }}$.

Replacing (10) in (9) and after some manipulations, the optimization problem (6) becomes

$$
\mathbf{u}_{k, j}=\arg \min _{\mathbf{u}_{k \mid k, j} \in \mathcal{U}_{k}} \max _{\boldsymbol{\omega}_{k \mid k, j} \in \mathbf{B}^{h \times\left(n_{x}+n_{y}\right)}} f\left(\mathbf{u}_{k \mid k, j}, \boldsymbol{\omega}_{k \mid k, j}\right)
$$

\footnotetext{
${ }^{3}$ Here the index $k+l \mid k$ is omitted and replaced by $k+l$ in order to
} simplify the notations. 
where $f\left(\mathbf{u}_{k \mid k, j}, \boldsymbol{\omega}_{k \mid k, j}\right)=\alpha_{1}+\alpha_{2} \boldsymbol{\omega}_{k \mid k, j}+\alpha_{3} \mathbf{u}_{k \mid k, j}+$

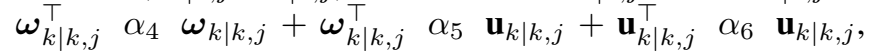
with $\quad \alpha_{1}=x_{k \mid k, j}^{\top} \sum_{l=0}^{h-1} A_{j}^{l^{\top}} Q A_{j}^{l} x_{k \mid k, j}, \quad \alpha_{2}=$ $2 x_{k \mid k, j}^{\top} \sum_{l=0}^{h-1} A^{l}{ }_{j}^{\top} Q \mathcal{A}_{l, j} \bar{F}, \quad \alpha_{3}=2 x_{k \mid k, j}^{\top} \sum_{l=0}^{h-1} A^{l^{\top}}{ }_{j}^{\top} Q \mathcal{A}_{l, j} \bar{B}$, $\alpha_{4}=\bar{F}^{\top} \sum_{l=0}^{h-1} \mathcal{A}_{l, j}^{\top} Q \mathcal{A}_{l, j} \bar{F}, \alpha_{5}=2 \bar{F}^{\top} \sum_{l=0}^{h-1} \mathcal{A}_{l, j}^{\top} Q \mathcal{A}_{l, j} \bar{B}$, $\alpha_{6}=\bar{F}^{\top} \sum_{l=0}^{h-1} \mathcal{A}_{l, j}^{\top} Q \mathcal{A}_{l, j} \bar{F}+\bar{R}$, and $\bar{B}=\operatorname{diag}(\underbrace{B, \ldots, B}_{h \text { times }})$, $\bar{F}=\operatorname{diag}(\underbrace{F, \ldots, F}_{h \text { times }})$ and $\bar{R}=\operatorname{diag}(\underbrace{R, \ldots, R}_{h \text { times }})$.

The function $f\left(\mathbf{u}_{k \mid k, j}, \boldsymbol{\omega}_{k \mid k, j}\right)$ is quadratic with respect to $\mathbf{u}_{k \mid k, j}$ and $\boldsymbol{\omega}_{k \mid k, j}$. In [22], it is shown that solving the Min-Max MPC problem (11) for all $\boldsymbol{\omega}_{k \mid k, j} \in \mathbf{B}^{h \times\left(n_{x}+n_{y}\right)}$ is equivalent to solve the following problem for all the vertices $\boldsymbol{\omega}_{k \mid k, j} \in \mathcal{V}_{\mathbf{B}^{h \times\left(n_{x}+n_{y}\right)}}$

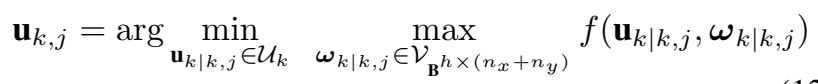

The problem (12) becomes a QP problem as follows

$$
\mathbf{u}_{k, j}=\arg \min _{\mathbf{u}_{k \mid k, j} \in \mathcal{U}_{k}} \tilde{f}\left(\mathbf{u}_{k \mid k, j}\right)
$$

such as $\tilde{f}\left(\mathbf{u}_{k \mid k, j}\right)$ is quadratic with respect to $\mathbf{u}_{k \mid k, j}$. In general, the constraints $x_{k} \in \mathbb{X}$ and $u_{k} \in \mathbb{U}$ are formulated such as $x_{\min } \leq x_{k} \leq x_{\max }$ and $u_{\min } \leq u_{k} \leq u_{\max }$. Finally, the problem (6) is rewritten in terms of the QP problem

$$
\begin{aligned}
& \underset{\mathbf{u}_{k \mid k, j}}{\min } \tilde{f}\left(\mathbf{u}_{k \mid k, j}\right) \\
& \text { subject to } \\
& {\left[\begin{array}{c}
\mathcal{A}_{l, j} \bar{B} \\
-\mathcal{A}_{l, j} \bar{B} \\
\mathcal{I}_{l} \\
-\mathcal{I}_{l}
\end{array}\right] \mathbf{u}_{k \mid k, j} \prec\left[\begin{array}{c}
b_{1} \\
b_{2} \\
u_{\max } \\
-u_{\min }
\end{array}\right]}
\end{aligned}
$$

for $l=1, \ldots, h$, with $b_{1}=x_{\max }-A_{j}^{l} x_{k \mid k}-\mathcal{A}_{l, j} \bar{F} \boldsymbol{\omega}_{k \mid k}$ and $b_{2}=-x_{\min }+A_{j}^{l} x_{k \mid k}+\mathcal{A}_{l, j} \bar{F} \boldsymbol{\omega}_{k \mid k}, \forall \boldsymbol{\omega}_{k \mid k} \in \mathcal{V}_{\mathbf{B}^{h \times\left(n_{x}+n_{y}\right)}}$ and $\mathcal{I}_{l}=\left[\begin{array}{lll}\mathbb{O}_{l-1, n_{u}} & \mathbb{1}_{1, n_{u}} & \mathbb{O}_{h-l, n_{u}}\end{array}\right]$.

\section{Illustrative EXAMPLE}

Consider the following LTI discrete-time system

$$
\left\{\begin{array}{c}
x_{k+1}=\left[\begin{array}{cc}
0.7 & 0.3 \\
0.6 & 0.7
\end{array}\right] x_{k}+\left[\begin{array}{c}
0.3 \\
0.2
\end{array}\right] u_{k}+ \\
+\left[\begin{array}{cccc}
0.05 & 0 & 0 & 0 \\
0 & 0.02 & 0 & 0
\end{array}\right] \omega_{k} \\
y_{k}=\left[\begin{array}{cc}
-2 & 1 \\
1 & 1
\end{array}\right] x_{k}+\left[\begin{array}{cccc}
0 & 0 & 0.01 & 0 \\
0 & 0 & 0 & 0.01
\end{array}\right] \omega_{k}
\end{array}\right.
$$

with $\left\|\omega_{k}\right\|_{\infty} \leq 1$. The value of $\omega_{k}$ is randomly generated. The initial state belongs to the ellipsoid $\mathcal{E}\left(\square_{2},\left[\begin{array}{ll}0 & 0\end{array}\right]^{\top}, 1\right)$. In this example, 4 models are considered. $M_{1}$ corresponds to the fault-free system, i.e. $A_{1}=A, B_{1}=B, C_{1}=C, E_{1}=$ $E$ and $F_{1}=F \cdot M_{2}$ models the system with a component fault: $A_{2}=\left[\begin{array}{cc}0.4 & 0.8 \\ 0.1 & 0.2\end{array}\right], B_{2}=B, C_{2}=C, E_{2}=E$ and $F_{2}=F . M_{3}$ corresponds to a patial actuator fault, with $A_{3}=A, B_{2}=\left[\begin{array}{c}0.15 \\ 0.1\end{array}\right], C_{3}=C, E_{3}=E$ and $F_{3}=F$. $M_{4}$ corresponds to the system having a partial fault in the second sensor: $A_{4}=A, B_{4}=B, C_{4}=\left[\begin{array}{cc}-2 & 1 \\ 0.5 & 0.5\end{array}\right]$, $E_{4}=E$ and $F_{4}=F$. The simulation length is $N=100$. The prediction horizon is $h=10$, the weighting matrices are $Q=$ $10 \cdot \square_{2}$ and $R=5$. The following constraints are considered on the state $x_{\min }=\left[\begin{array}{ll}-1 & -1\end{array}\right]^{\top}, x_{\max }=\left[\begin{array}{ll}1 & 1\end{array}\right]^{\top}$, and on the input signal $u_{\min }=-0.8$ and $u_{\max }=0.8$. The simulated faults are described in Table I.

TABLE I

SIMULATED FAULT SCENARIO

\begin{tabular}{lc} 
Fault description & Time interval (samples) \\
\hline $50 \%$ fault in actuator & $10-20$ \\
$50 \%$ fault in sensor & $50-60$
\end{tabular}

Figures 1 and 2 illustrate the bounds of $x_{1}$ and $x_{2}$ after 100 iterations. The solid blue lines represent the bounds obtained by Algorithm 1. The red stars represent the real state of the system (situated inside the estimated bounds). The state estimation is guaranteed despite the presence of the considered faults, however the bounds of the estimation set are larger when faults occur (compared to a fault-free time intervals). In Figure 3, the control $u_{k}$ is represented. The constraint $u_{\min } \leq u_{k} \leq u_{\max }$ is satisfied.

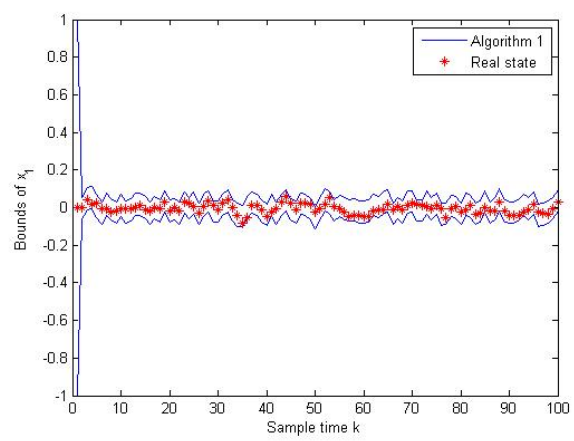

Fig. 1. Bounds of $x_{1}$

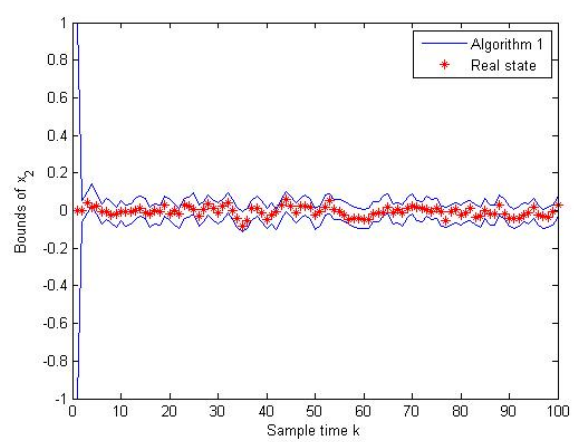

Fig. 2. Bounds of $x_{2}$ 

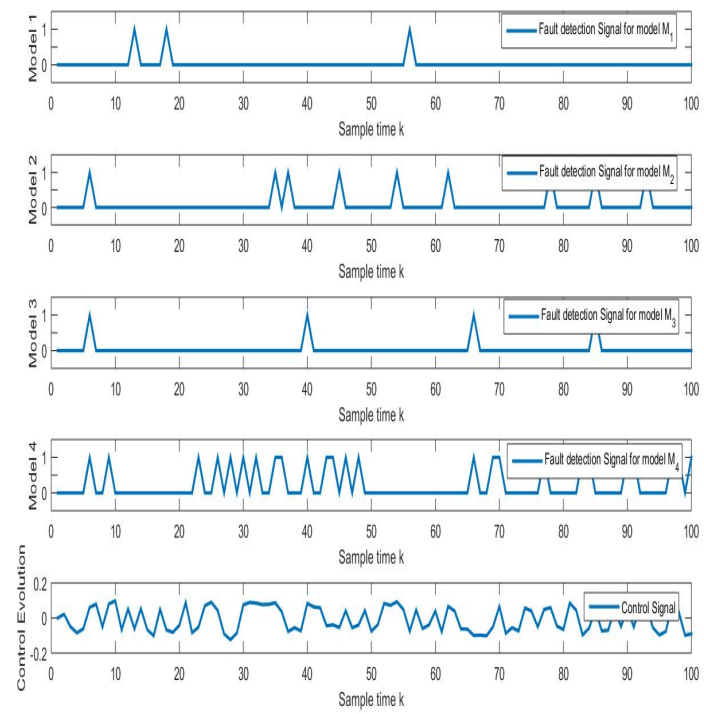

Fig. 3. Evolution of the control $u$ and the fault signals

Figure 3 shows also the fault signal obtained by models $M_{1}, M_{2}, M_{3}$ and $M_{4}$, respectively. When the fault signal is equal to 0 (respectively 1 ), the model $M_{i}$ is compatible (respectively incompatible) with the measurements. Effectively, the model $M_{1}$ corresponding to the fault-free case system is compatible with the measurement when there is no fault. Even if for the considered actuator fault (between 10-20 samples), the models $M_{2}, M_{3}$ and $M_{4}$ are compatible with the measurements, the optimal model chosen by the Min-Max MPC is $M_{3}$. In a similar way, the model $M_{4}$ is chosen (between the compatible models $M_{3}$ and $M_{4}$ ) the optimal model for the considered sensor fault. This confirms the performance of Algorithm 1.

\section{CONCLUSION}

A new Fault Fault Tolerant Control method based on Multiple Models for linear systems with bounded perturbations and measurement noises has been proposed. Despite the presence of simultaneous faults on component, actuators and/or sensors, the proposed algorithm allows to estimate the state of the system in a set-membership framework. A Min-Max MPC based on the ellipsoidal state estimation has been used in order to choose the best model for the estimation within faulty situations, while minimizing the system energy for the worst perturbations. An example illustrates the effectiveness of the proposed method.

\section{REFERENCES}

[1] R. J. Patton, P. M. Frank, and R. N. Clark, "Issues of fault diagnosis for dynamic systems," Springer-Verlag London Ltd, 2000.

[2] M. Blanke, M. Kinnaert, J. Lunze, and M. Staroswiecki, "Diagnosis and Fault-Tolerant Control," Springer-Verlag, 2003.
[3] P. S. Maybeck, "Multiple model adaptive algorithms for detecting and compensating sensor and actuator/surface failures in aircraft flight control systems," International Journal of Robust and Nonlinear Control, vol. 9(14), pp. 1051-1070, 1999.

[4] J. Ru and X. R. Li, "Variable-structure multiple-model approach to fault detection, identification, and estimation," IEEE Transaction on Control System Technology, 2008.

[5] A. Banerjee, Y. Arkun, B. Ogunnaike, and R. Pearson, "H-infinity Control of Nonlinear Processes using Multiple Linear Models," in Proc. of European Control Conference, Rome, Italy, 1995.

[6] Y. Zhang and X. R. Li, "Detection and diagnosis of sensor and actuator failures using IMM estimator," IEEE Transactions on Aerospace and Electronic Systems, vol. 34(4), pp. 1293-1313, 1998.

[7] G. G. Yen and L. W. Ho, "Online multiple-model-based fault diagnosis and accommodation," IEEE Transactions on Industrial Electronics, vol. 50(2), pp. 296-312, 2003.

[8] V. G. Verma, R. Simmons, and S. Thrun, "Real-time fault diagnosis," IEEE Robotics and Automation Magazine, vol. 11(2), pp. 56-66, 2004.

[9] G. Ducard and H. P. Geering, "Efficient nonlinear actuator fault detection and isolation system for unmanned aerial vehicles," Journal of Guidance, Control, and Dynamics, vol. 31(1), pp. 225-237, 2008.

[10] R. Hallouzi, V. Verdult, R. Babuska, and M. Verhaegen, "Fault detection and identification of actuator faults using linear parameter varying models," in Proc. of the 16th Triennial World Congress IFAC, Prague, Czech Republic, 2005.

[11] M. Rodrigues, D. Teilliol, S. Aberkane, and D. Sauter, "Fault tolerant control design for polytopic LPV systems," International Journal of Applied Mathematics and Computer Science, vol. 17(1), 2007.

[12] M. Witczak, L. Dziekan, V. Puig, and J. Korbicz, "An integrated design strategy for fault identification and fault-tolerant control for TakagiSugeno fuzzy systems," in Proc. of the 17th World Congress of the International Federation of Automatic Control, Seoul, Korea, 2007.

[13] H. Hamdi, M. Rodrigues, C. Mechmeche, and N. B. Braiek, "Robust Fault Detection and estimation for descriptor systems based on MultiModels concept," International Journal of Control, Automation, and Systems, IJCAS, vol. 10(6), pp. 1260-1266, 2012.

[14] B. Marx, D. Koenig, and J. Ragot, "Design of observers for TakagiSugeno nonlinear descriptor systems with unknown inputs and application to fault diagnosis," IET Control Theory Application, vol. 1, pp. 1487-1495, 2007.

[15] R. Hallouzi, M. Verhaegen, R. Babuska, and S. Kanev, "Model weight and state estimation for Multiple Model systems applied to Fault Detection and Identification," in Proc. of the 14th IFAC Symposium on System Identification, Newcastle, Australia, 2006.

[16] M. A. Medina, D. Theilliol, and D. Sauter, "Simultaneous fault diagnosis and robust activation function estimation of linear multiple-models considering the bounded modelling uncertainty," in Mediterranean Conference on Control and Automation, 2005, Limassol, Cyprus.

[17] F. C. Schweppe, "Recursive state estimation: Unknown but bounded errors and system inputs," IEEE Trans. Automat. Contr., vol. 13(1), pp. 22-28, 1968.

[18] D. P. Bertsekas and I. B. Rhodes, "Recursive state estimation for a setmembership description of uncertainty," IEEE Trans. Automat. Contr. vol. 16(2), pp. 117-128, 1971.

[19] F. L. Chernousko, State estimation for dynamic systems. Boca Raton: CRC Press, 1994.

[20] E. Fogel and Y. F. Huang, "On the value of information in system identification-bounded noise case," Automatica, vol. 18, 1982.

[21] S. Ben Chabane, C. Stoica Maniu, T. Alamo, E. Camacho, and D. Dumur, "A new approach for guaranteed ellipsoidal state estimation," in Proc. of the 19th World Congress IFAC, Cape Town, 2014.

[22] T. Alamo, D. R. Ramirez, and D. M. de la Pena, "Min-Max MPC using a tractable QP problem," in Proc. of the 49th IEEE CDC and $E C C, 2005$, Seville, Spain.

[23] T. Alamo, J. M. Bravo, and E. F. Camacho, "Guaranteed state estimation by zonotopes," Automatica, vol. 41, pp. 1035-1043, 2005.

[24] V. T. H. Le, C. Stoica, T. Alamo, E. F. Camacho, and D. Dumur, "Zonotopic guaranteed state estimation for uncertain systems," Automatica, vol. 49(1), pp. 3418-3424, 2013.

[25] C. Durieu, E. Walter, and B. Polyak, "Multi-input multi-output ellipsoidal state bounding," Journal of optimization theory and applications, vol. 111(2), pp. 273-303, 2001. 